\title{
Globe
}

Revue internationale d'études québécoises

\section{Alain Laberge, avec la collaboration de Jacques Mathieu et Lina Gouger, Portraits de campagnes : la formation du monde rural laurentien au XVIII siècle, Québec, Presses de l’Université Laval, 2010}

Maxime Morin

Volume 16, numéro 1, 2013

URI : https://id.erudit.org/iderudit/1018188ar

DOI : https://doi.org/10.7202/1018188ar

Aller au sommaire du numéro

Éditeur(s)

Globe, Revue internationale d'études québécoises

ISSN

1481-5869 (imprimé)

1923-8231 (numérique)

Découvrir la revue

Citer ce compte rendu

Morin, M. (2013). Compte rendu de [Alain Laberge, avec la collaboration de Jacques Mathieu et Lina Gouger, Portraits de campagnes : la formation du monde rural laurentien au XVIII ${ }^{e}$ siècle, Québec, Presses de l’Université Laval, 2010]. Globe, 16(1), 214-217. https://doi.org/10.7202/1018188ar d'utilisation que vous pouvez consulter en ligne.

https://apropos.erudit.org/fr/usagers/politique-dutilisation/ 
d'anecdotes fort intéressantes sur la prohibition à Montréal - pensons aux différentes pièces cadenassées au sein d'une même maison de jeux, une véritable farce (p. 57-58) -, tandis que la seconde est fort bien faite. Dans ces deux chapitres courts, synthétiques et bien écrits, Magaly Brodeur montre bien toutes les difficultés rencontrées par la Ville de Montréal pour boucler son budget, et que la célèbre «taxe volontaire» de Jean Drapeau n'était que la dernière des tentatives - la plus aboutie, aussi - des hommes politiques de la métropole pour légaliser le jeu et récupérer cette source de revenus. Cela dit, le passage du mémoire à l'ouvrage pose un problème majeur qui ne peut être négligé.

Alexandre Turgeon

Université Laval

\section{Alain Laberge, avec la collaboration de Jacques Mathieu et Lina Gouger, \\ Portraits de campagnes : la formation du monde rural laurentien au XVIII siècle, Québec, Presses de l'Université Laval, 2010.}

Professeur au Département d'histoire de l'Université Laval, Alain Laberge a réalisé cet ouvrage avec la collaboration des historiens Jacques Mathieu et Lina Gouger. Cette étude de la formation du monde rural laurentien s'appuie sur les découpages fonciers et la spatialisation du peuplement. Laberge y distingue les environnements de base du paysage seigneurial laurentien par la mise en rapport des éléments qui l'ont façonné. Loin d'en proposer un portrait simplifié, Laberge démontre qu'il constitue à toutes les échelles une véritable mosaïque : "Ce terme, assurément, correspond parfaitement au paysage humanisé de la vallée du Saint-Laurent rurale à cette époque» (p. 139). Ce constat repose principalement sur l'analyse des aveux et dénombrements produits entre 1723 et 1745 dans 177 seigneuries du corridor rural laurentien. L'aveu et dénombrement, une déclaration faite par le seigneur à la demande de l'autorité royale, identifie le seigneur, localise la seigneurie et en précise les dimensions, dresse l'inventaire systématique des parcelles de terre découpées, puis rend compte de l'exploitation du domaine seigneurial et des censives concédées. Si l'utilisation de cette série documen- 
taire favorise une analyse synchronique du monde rural vers 1725 , époque où sont produits la plupart de ces documents, Laberge inscrit cet instantané dans le long processus de formation des campagnes laurentiennes.

L'ouvrage débute par une reconstitution du paysage seigneurial. Laberge y présente les principales caractéristiques de l'espace seigneurial en examinant le rythme et les types de concessions, les mutations du territoire seigneurial et la morphologie des seigneuries concédées. L'écoumène formé par le territoire concédé dans la vallée laurentienne prend forme autour de Québec, Trois-Rivières et Montréal. Alors que les premières seigneuries concédées sont souvent de taille excessive, la morphologie des seigneuries concédées après 1663 tend vers un rectangle d'un front de deux lieues ou moins sur l'axe laurentien dans $80 \%$ des cas et d'une profondeur qui reste fort variable. Dès lors, le lecteur est confronté à la diversité du paysage seigneurial.

Laberge observe ensuite les processus d'occupation du territoire rural, que ce soit les conditions, l'ancienneté et l'intensité de cette occupation, l'extension de l'écoumène, les schémas d'occupation ou la morphologie des seigneuries, paroisses et censives. Il souligne que plusieurs dynamiques parallèles forgent les campagnes laurentiennes à des rythmes différents. Dans l'ensemble, l'axe laurentien demeure un facteur déterminant dans le processus de concession des seigneuries et des censives. L'intensité et l'ancienneté de l'occupation plus marquée à Québec vers 1725 ne cachent pas l'ascension de Montréal comme plus important foyer d'occupation rural de la colonie. Le modèle d'occupation littorale sur la devanture des fiefs caractérise la majorité des terres en censive même si leur morphologie n'est pas uniforme.

L'exploitation du territoire seigneurial n'est d'ailleurs pas plus homogène que son occupation, comme le remarque Laberge à propos de la répartition des superficies exploitées en prairies ou en labours, de la présence marquée des fronts pionniers dans l'écoumène seigneurial, des banlieues comme zones intenses de développement agricole ou des usages particuliers des terres exploitées. Si la diversité des réalités est encore au menu, quelques constats révisent le schéma de la seigneurie-modèle à cette époque. Par exemple, seulement $20 \%$ des seigneuries comprennent une commune contrairement au caractère omniprésent que revêt généralement cet élément du paysage seigneurial dans l'historiographie. À terme, l'observation des processus de concession, d'occupation et d'exploitation du territoire laurentien à l'époque des aveux et dénombrements confirme que "[r]ien ne serait plus éloigné de la réalité que d'affirmer que la vallée du Saint-Laurent constitue un paysage uniforme sous le Régime français» (p. 73). 
L'hétérogénéité du paysage seigneurial se vérifie aussi lorsqu'il est question des seigneurs et des censitaires dans la seconde partie de l'ouvrage. Laberge traite d'abord de la propriété seigneuriale où il fait le point sur la répartition sociale, la provenance, le contrôle et la composition de la propriété seigneuriale dans le monde rural laurentien à l'époque des aveux et dénombrements. Exposant la diversité des scénarios possibles, il montre que la propriété seigneuriale constitue un enjeu qui repose sur des motivations d'élévation sociale chez les seigneurs roturiers, comme chez les nobles qui cherchent en outre à consolider leur position par des stratégies matrimoniales et successorales. En s'intéressant ensuite aux interventions des seigneurs dans l'aménagement, le développement et la gestion de leurs fiefs, Laberge précise l'enjeu économique que représentent leurs seigneuries. Il compare les différents stades de développement des domaines et des terres seigneuriales, y relève la présence de bâtiments et de terres voués aux services, puis en dresse un portrait en fonction du statut social des seigneurs. Règle générale, les terres réservées aux seigneurs représentent un enjeu économique important, autant sur le plan de la production agricole qu'afin de doter les domaines des principaux équipements nécessaires aux censitaires. Les seigneurs sont des acteurs clés dans le processus d'emprise du sol et, même si leurs revenus restent souvent un facteur incertain vers 1725 , les fronts pionniers progressent et la conjoncture économique favorable qui s'installe les encourage à augmenter la production.

L'auteur se tourne enfin vers les censitaires afin d'en reconstituer divers profils. En portant attention aux censitaires non paysans d'une part, il note que les censives ne sont pas l'apanage des habitants puisqu'elles contribuent aussi à la consolidation de la propriété foncière des seigneurs. En dressant une typologie des censitaires paysans à partir des aveux et dénombrements d'autre part, il établit que la variabilité de la condition paysanne résulte de deux processus complémentaires. Elle est le produit à la fois des écarts entre les générations de paysans et d'une capacité de production et de reproduction familiale inégale chez les paysans. Ainsi, le dynamisme interne du monde rural laurentien repose sur l'action simultanée des seigneurs et des censitaires dont les motivations et les aspirations respectives sont distinctes.

En définitive, une multitude de réalités concomitantes font du corridor rural laurentien une mosaïque en lente mais constante évolution. Par un jeu d'échelles, Laberge génère non pas un, mais bien plusieurs portraits de campagnes. Délaissant une vision synthétique atténuant les particularités régionales et seigneuriales qui émergent de son analyse, l'auteur nuance les visions misérabilistes ou triomphalistes traditionnellement 
exprimées au sujet de la colonisation de la vallée laurentienne sous le Régime français. C'est là que réside la principale contribution de cet ouvrage. Laberge étudie le processus d'emprise du sol autant dans sa dimension spatiale que dans une perspective de longue durée et cela se traduit par un impressionnant travail de cartographie et d'analyse quantitative. L'ouvrage, d'une très belle facture, mise aussi sur la reproduction de pièces d'archives et une iconographie variée, de sorte qu'il fait belle figure dans la collection des Atlas historiques du Québec. Un seul bémol s'il en est un, une version papier de l'ouvrage aurait été appréciée, lui qui est uniquement diffusé en version électronique par les Presses de l'Université Laval. Ce format sert toutefois bien la cartographie de l'ouvrage dont le degré de détails nécessite des agrandissements depuis un logiciel de visualisation. Ainsi le lecteur pourra-t-il aussi apprécier de visu l'un ou l'autre des portraits de campagnes qui y sont dépeints.

Maxime Morin

Université Laval 